\title{
Magnetostrictive vibration damper and energy harvester for rotating machinery
}

\author{
Zhangxian Deng $^{a}$, Vivake M. Asnani ${ }^{b}$, Marcelo J. Dapino $^{a}$ \\ ${ }^{a}$ NSF I/UCRC on Smart Vehicle Concepts, Department of Mechanical and Aerospace \\ Engineering, The Ohio State University, Columbus, OH, 43210, USA \\ ${ }^{b}$ NASA Glenn Research Center, Cleveland, OH, 44135, USA
}

\begin{abstract}
Vibrations generated by machine driveline components can cause excessive noise and structural damage. Magnetostrictive materials, including Galfenol (iron-gallium alloys) and Terfenol-D (terbium-irondysprosium alloys), are able to convert mechanical energy to magnetic energy. A magnetostrictive vibration ring is proposed, which generates electrical energy and dampens vibration, when installed in a machine driveline. A 2D axisymmetric finite element (FE) model incorporating magnetic, mechanical, and electrical dynamics is constructed in COMSOL Multiphysics. Based on the model, a parametric study considering magnetostrictive material geometry, pickup coil size, bias magnet strength, flux path design, and electrical load is conducted to maximize loss factor and average electrical output power. By connecting various resistive loads to the pickup coil, the maximum loss factors for Galfenol and Terfenol-D due to electrical energy loss are identified as 0.14 and 0.34 , respectively. The maximum average electrical output power for Galfenol and Terfenol-D is $0.21 \mathrm{~W}$ and $0.58 \mathrm{~W}$, respectively. The loss factors for Galfenol and Terfenol-D are increased to 0.59 and 1.83, respectively, by using an L-C resonant circuit.
\end{abstract}

Keywords: Magnetostrictive materials, vibration damper, energy harvester, COMSOL Multiphysics

\section{INTRODUCTION}

Vibrations generated by machine driveline components can cause excessive noise and structural damage. Piezoelectric materials are able to transduce mechanical energy to electrical energy; thus they can be implemented as passive structural dampers. A PZT device was proposed which applies damping in a driveline to reduce unwanted vibration and noise. ${ }^{1}$ The device has the appearance of a metal ring, as shown in Figure 1.

Magnetostrictive materials, which couple mechanical and magnetic energy, have been widely implemented in damping and energy harvesting applications. ${ }^{2-7}$ Many magnetic materials exhibit magnetomechanical coupling, but only a few materials containing rare earth elements show significant or giant magnetomechanical coupling effects. Terbium-iron-dysprosium alloys, known as Terfenol-D, are able to generate $1600 \mathrm{ppm}$ magnetostriction, but a high magnetic field $(160 \mathrm{kA} / \mathrm{m})$ is required to saturate this material. ${ }^{8}$ Iron-gallium alloys, known as Galfenol, are a recent class of magnetostrictive materials that exhibit moderate magnetostriction $(400 \mathrm{ppm})$ and high magnetization $(1200 \mathrm{kA} / \mathrm{m})$ at low magnetic fields. ${ }^{9}$ Figure 2 shows a new vibration ring based on magnetostrictive rods. The rods are arranged radially around the circumference, and are made of Terfenol-D or Galfenol. With this configuration, the vibration ring is able to convert radial vibration energy to electrical energy, thus creating a vibration damping effect. Unlike Terfenol-D and piezoelectric materials, Galfenol has a mechanical robustness

Further author information: (Send correspondence to M.J.D)

Z.D.: E-mail: deng.92@osu.edu, Telephone: 1-614-886-4687

M.J.D.: E-mail: dapino.1@osu.edu, Telephone: 1-614-688-3689

Industrial and Commercial Applications of Smart Structures Technologies 2015,

edited by Kevin M. Farinholt, Steven F. Griffin, Proc. of SPIE Vol. 9433, 94330C

(c) 2015 SPIE · CCC code: 0277-786X/15/\$18 - doi: 10.1117/12.2085566

Proc. of SPIE Vol. 9433 94330C-1 


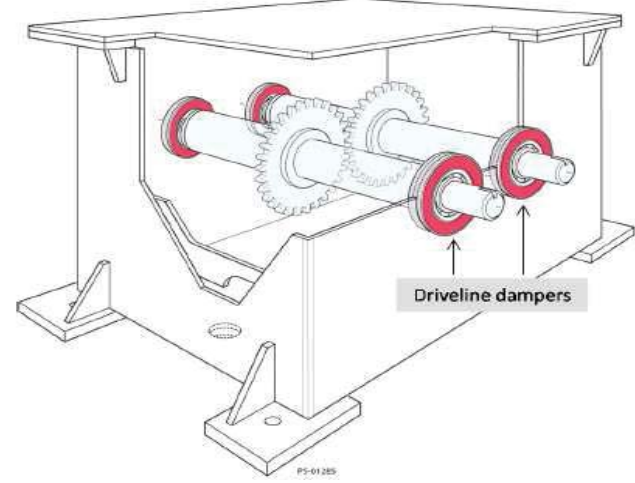

(a)

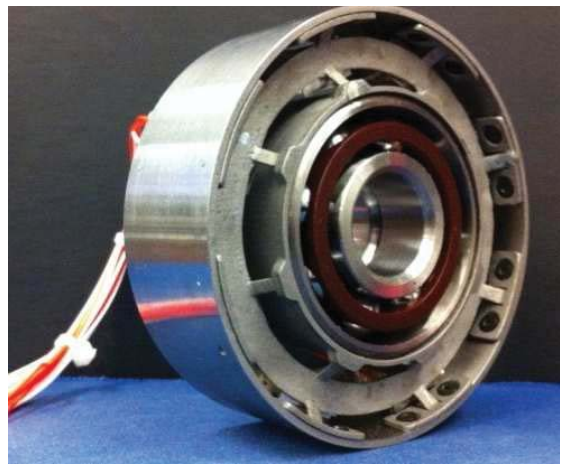

(b)

Figure 1. (a) Vibration ring locations in a typical transmission; (b) NASA's piezoelectric vibration ring. ${ }^{1}$

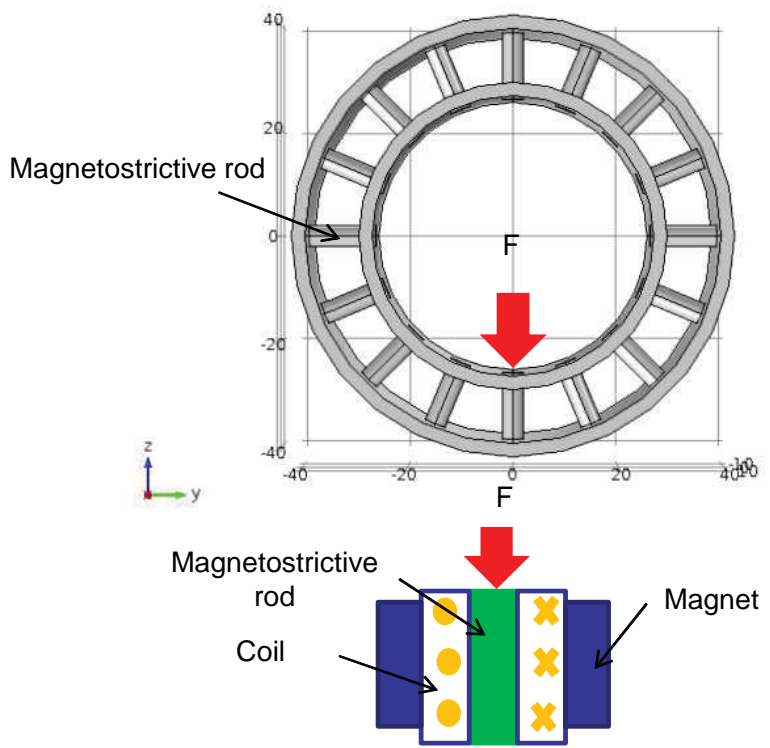

Figure 2. Compression spoke design of a magnetostrictive vibration ring. Terfenol-D has a tensile strenght of about $28 \mathrm{MPa}$, thus it must be operated in compression. The Terfenol-D rod is press fit between the two rings. Galfenol can be threaded into the rings, since it has a tensile strength of about $500 \mathrm{MPa}$ and good resistance to torsion and impact. 
Table 1. Comparison of Terfenol-D and Galfenol. ${ }^{6,12-14}$

\begin{tabular}{c|c|c}
\hline Material & Terfenol-D & Galfenol \\
\hline Composition & $\mathrm{Tb}_{\mathrm{x}} \mathrm{D}_{1-\mathrm{x}} \mathrm{Fe}_{2}(\mathrm{x} \approx 0.3)$ & $\mathrm{Fe}_{1-\mathrm{x}} \mathrm{Ga}_{\mathrm{x}}(\mathrm{x}=0.12-0.33)$ \\
Conductivity $(\mathrm{S} / \mathrm{m})$ & $1.72 \times 10^{6}$ & $5.9610^{6}$ \\
Relative permeability & $3-10$ & $1-700$ \\
Saturated Young's modulus $(\mathrm{GPa})$ & 115 & $60-80$ \\
Density $\left(\mathrm{kg} / \mathrm{m}^{3}\right)$ & 9250 & 7870 \\
Tensile strength $(\mathrm{MPa})$ & 28 & 500 \\
Static hysteresis loss per cycle $\left(\mathrm{kJ} / \mathrm{m}^{3}\right)$ & 23 & 2.1 (Constant field biasing) \\
\hline
\end{tabular}

similar to steel, and thus can be machined, welded, and formed. Without undergoing permanent depolarization, magnetostrictive materials also maintain significant magnetomechanical coupling over a broad temperature range. ${ }^{10,11}$ Table 1 compares the mechanical and magnetic properties of Terfenol-D and Galfenol.

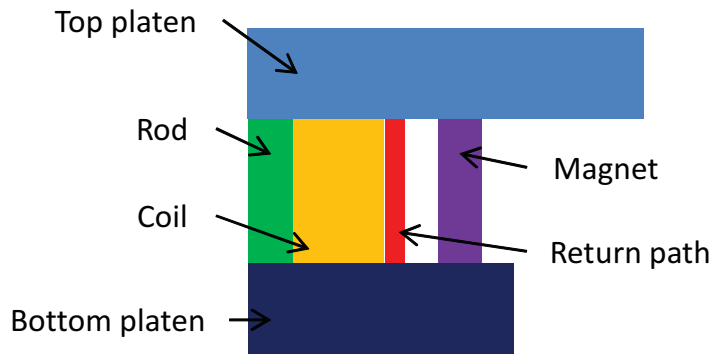

(a)

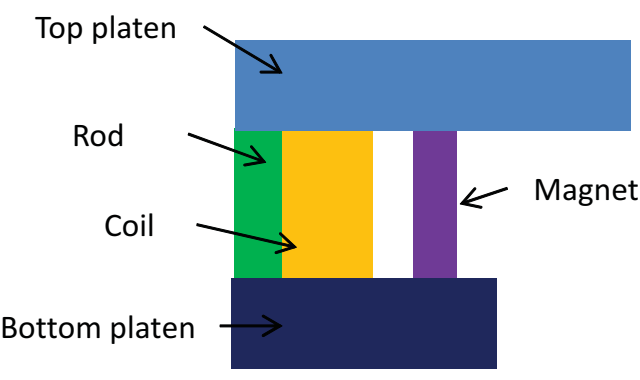

(b)

Figure 3. (a) Geometry in COMSOL Multiphysics for Galfenol analysis; (b) geometry in COMSOL Multiphysics for Terfenol-D analysis.

Instead of modeling the entire vibration ring directly, this study investigates the damping and energy harvesting properties of the Galfenol and Terfenol-D rods, which are the building blocks of the design. Two dimensional (2D) axisymmetric models, as shown in Figure 3, were created in COMSOL Multiphysics. The length of the rods is $10 \mathrm{~mm}$. The top and bottom platens are made of stainless steel A430. The return path is made of 1018 low carbon steel. A $750 \mathrm{~Hz}, 280 \mathrm{~N}$ amplitude sinusoidal force (the target vibration source) is applied on the top surface of the rods, while their bottom surface is fixed. Cylindrical Alnico magnets (Grade 8) with a remanent flux density of $0.6 \mathrm{~T}$ are used to generate a bias magnetic field through the magnetostrictive rods. The 2D axisymmetric model incorporates magnetic, mechanical, and electrical dynamics. Based on the proposed model, the performance of the Galfenol and Terfenol-D rods is investigated in terms of loss factor due to electrical energy loss $\eta$ and average electrical output power $\bar{P}$. A parametric study for rod geometries, coil size, and magnetic flux path is also presented.

\section{PERFORMANCE CONSIDERATIONS}

\subsection{Loss factor $\eta$}

Loss factor is a common measure of a material's intrinsic damping. It is defined for a steady-state oscillation as ${ }^{15}$

$$
\eta=\frac{D}{2 \pi W}
$$


where $D$ is the energy dissipated and $W$ is the energy that is returned to the input with each cycle. For magnetostrictive components, there are several loss mechanisms, including hysteresis loss, mechanical damping, eddy current loss, and dissipation of generated electrical energy. In this study, the only loss considered is the latter. Therefore, the electrical energy loss per cycle, $E_{l o s s}$, is maximized in order to maximize the loss factor.

In the frequency domain, the sinusoidal input force $F^{*}$ with a frequency $f_{0}$ can be written as

$$
F^{*}=F_{0} e^{j \phi_{F}},
$$

where $F_{0}$ is the force amplitude and $\phi_{F}$ is the phase of the signal. Since magnetostrictive materials are highly nonlinear, the tip displacement due to $F^{*}$ consists of a fundamental component $S^{*}$ and higher harmonics. The loss factor in this study is calculated for the fundamental frequency of the response using the Fast Fourier Transform (FFT) method. ${ }^{16}$ The fundamental component of the displacement is defined as

$$
S^{*}=S_{0} e^{j \phi_{S}},
$$

where $S_{0}$ and $\phi_{S}$ are the displacement amplitude and phase, respectively. Following ASTM D5992$96(2011),{ }^{16}$ the loss factor is calculated from the phase difference as

$$
\eta=\tan \left(\phi_{F}-\phi_{S}\right) .
$$

The ASTM standard method for calculation loss factor is employed so that the results are comparable with commonly available data on passive material damping, such as for rubber or metals.

\subsection{Average electrical output power $\bar{P}$}

Figure 4 shows the equivalent circuit for the electrical system, including the resistance and inductance of the pickup coil and electrical shunt components. Both purely resistive and parallel resistive-capacitive shunts are analyzed in this study. The average electrical output power $\bar{P}$ on the resistor $R_{L}$ quantifies the harvested energy and it is calculated as

$$
\bar{P}=\int_{0}^{T} \frac{V_{L}(t)^{2}}{R_{L}} d t
$$

where $V_{L}(t)$ is the voltage on the resistive load and $T$ is the period of the vibration.

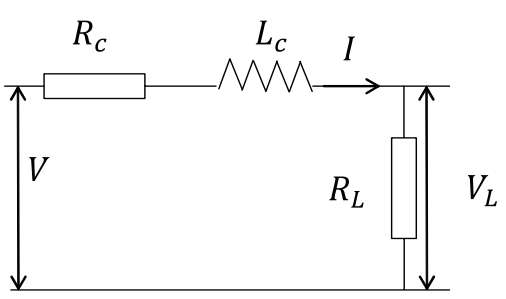

(a)

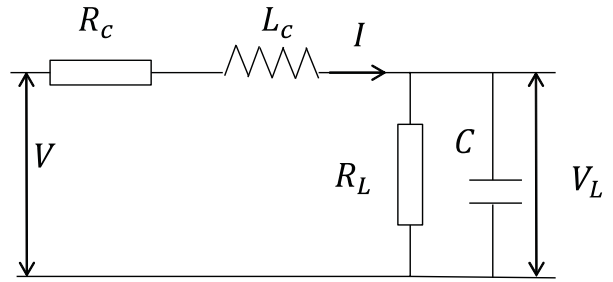

(b)

Figure 4. (a) Resistive load; (b) capacitive load. 


\subsection{Purely resistive load}

A previous study ${ }^{17}$ showed that $\bar{P}$ for Figure 4(a) is maximum when

$$
R_{L}=\sqrt{R_{c}^{2}+\left(L_{c} \omega\right)^{2}}
$$

where $R_{c}$ is the coil resistance, $L_{c}$ is the coil inductance, and $\omega$ is the radial frequency of the vibration. The electrical energy loss per cycle $E_{\text {loss }}$ is

$$
\begin{aligned}
E_{\text {loss }} & =\int_{0}^{T} I(t)^{2}\left(R_{c}+R_{L}\right) d t \\
& =\frac{V_{a m p}^{2} T}{2} \frac{R_{c}+R_{L}}{\left(R_{c}+R_{L}\right)^{2}+\left(L_{c} \omega\right)^{2}},
\end{aligned}
$$

where $I(t)$ is the current through the coil and $V_{a m p}$ is the amplitude of the voltage generated by the coil. To maximize $E_{\text {loss }}, R_{L}$ should satisfy

$$
R_{L}=\left\{\begin{array}{lr}
L_{c} \omega-R_{c} & \left(L_{c} \omega>R_{c}\right) \\
0 & \left(L_{c} \omega \leq R_{c}\right)
\end{array}\right.
$$

The maximum electrical energy loss is

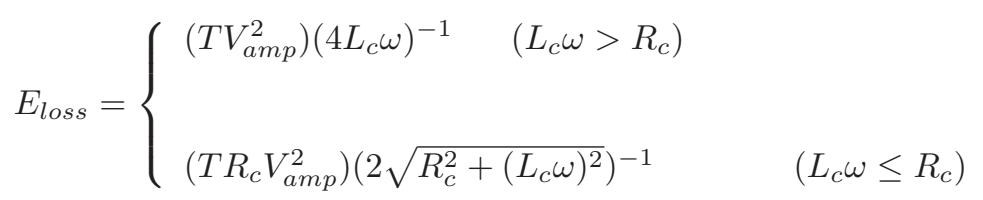

Equations (6) and (8) show that the maximum output power and the maximum loss factor do not happen at the same load conditions. To bring the two optimal resistances closer, either $R_{c}$ should be

reduced or $L_{c}$ needs to be increased. Equation (9) shows that increasing $L_{c}$ will reduce the maximum energy loss which is detrimental. On the other hand, the coil resistance $R_{c}$ can be reduced by using copper wires with a larger cross section. However, the larger wire size limits the number of turns per layer, thus reducing $V_{a m p}$. This tradeoff will be investigated in future work.

\subsection{Resistive-capacitive load}

Previous studies showed that energy dissipation can be improved by connecting a capacitor in parallel with the resistive load (Figure $4(\mathrm{~b})) .{ }^{3}$ The average power dissipated by the pickup coil is

$$
\begin{aligned}
P_{c} & =\frac{1}{T} \int_{0}^{T} I^{2}(t) R_{c} d t \\
& =\frac{\left|V_{a m p}\right|^{2}}{2} \frac{\left[\left(C R_{L} \omega\right)^{2}+1\right] R_{c}}{\left(R_{L}+R_{c}-L_{c} R_{L} C \omega^{2}\right)^{2}+\left(R_{L} R_{c} C+L_{c}\right)^{2} \omega^{2}} .
\end{aligned}
$$

The average electrical output power is

$$
\begin{aligned}
\bar{P} & =\frac{1}{T} \int_{0}^{T} \frac{V_{L}^{2}(t)}{R_{L}} d t \\
& =\frac{\left|V_{a m p}\right|^{2}}{2} \frac{R_{L}}{\left(R_{L}+R_{c}-L_{c} R_{L} C \omega^{2}\right)^{2}+\left(R_{L} R_{c} C+L_{c}\right)^{2} \omega^{2}} .
\end{aligned}
$$


According to a previous study, ${ }^{17}$ the maximum $\bar{P}$ is

$$
\bar{P}=\frac{V_{a m p}^{2}}{8 R_{c}},
$$

when

$$
C=\frac{L_{c}}{R_{c}^{2}+\left(L_{c} \omega\right)^{2}} \text { and } R_{L}=R_{c}+\frac{L_{c}^{2} \omega^{2}}{R_{c}} .
$$

The total electrical energy loss per cycle $E_{\text {loss }}$ is

$$
\begin{aligned}
E_{\text {loss }} & =\left(P_{c}+\bar{P}\right) T \\
& =\frac{\left|V_{a m p}\right|^{2} T}{2} \frac{\left(R_{L} C \omega\right)^{2} R_{c}+\left(R_{c}+R_{L}\right)}{\left(R_{L}+R_{c}-L_{c} R_{L} C \omega^{2}\right)^{2}+\left(R_{L} R_{c} C+L_{c}\right)^{2} \omega^{2}},
\end{aligned}
$$

which is maximum when $C=1 /\left(L_{c} \omega^{2}\right)$ and $R_{L}=+\infty$. In other words, the maximum energy loss is achieved when $R_{L}$ is disconnected and the L-C circuit has a natural frequency that is the same as the driving frequency. The capacitor connected in parallel compensates for the electrical impedance due to the coil inductance and improves the loss factor. However, its performance degrades when the driving frequency shifts away from the L-C resonance.

\section{NUMERICAL COMPARISON OF GALFENOL AND TERFENOL-D}

\subsection{Pickup coil size}

The pickup coil is made of AWG 36 copper wire. The flux density through the rod is assumed to be a $750 \mathrm{~Hz}, 0.2 \mathrm{~T}$ sinusoidal wave, and the pickup coil is short-circuited. The induced voltage on the pickup coil increases with the number of layers until the outer layers become insensitive to the flux density change on the rod. On the other hand, the coil resistance increases rapidly with respect to the coil size. Hence, an optimal number of layers exists to maximize the average electrical output power of the coil. Figure 5 shows that a pickup coil with 29 layers dissipates the maximum amount of energy.

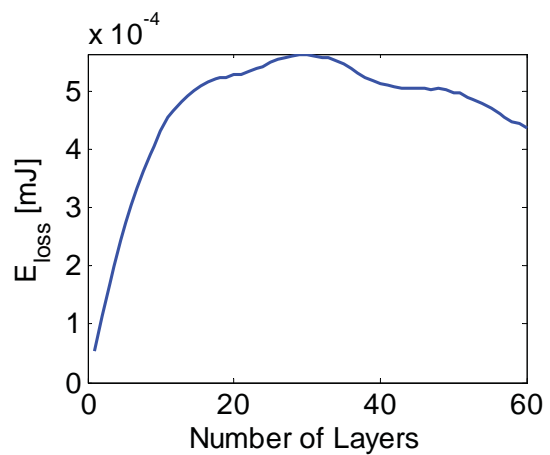

Figure 5. Energy dissipated by the pickup coil per cycle with respect to the number of layers in the pickup coil.

\subsection{Flux path}

The permeability of Terfenol-D is close to that of air, so part of the flux generated by the permanent magnets leaks through air. The magnetic field through the Terfenol-D spoke is

$$
H_{T}=\frac{\Phi}{A_{r}+\left(\mu_{0} / \mu_{T}\right) A_{A}}
$$


where $\Phi$ is the flux generated by the permanent magnet, $A_{r}$ is the cross-sectional area of the magnetostrictive rod, $\mu_{0}$ is the permeability of air, $\mu_{T}$ is the permeability of the Terfenol element, and $A_{A}$ is the cross-sectional area of air. The term $\mu_{T}$ changes with respect to applied stress, but the variation is relatively small (Table 1). Hence, the bias magnetic field through the Terfenol-D rod is nearly constant.

Galfenol has a relatively high magnetic permeability, so almost all the magnetic flux flows through the Galfenol cross section. The magnetic field through the Galfenol rod is

$$
H_{G}=\frac{\Phi}{A_{r} \mu_{G}},
$$

where $\mu_{G}$ is the permeability of Galfenol. According to Table $1, \mu_{G}$ has a large variation with respect to applied stress, thus the magnetic field $H_{G}$ changes significantly during operation. Previous research ${ }^{18,19}$ showed that the maximum magnetomechanical coupling is achieved when the magnetic field through the magnetostrictive materials is constant.

To prevent the magnetic field in the Galfenol rod from varying, a flux path is added in parallel to the Galfenol rod, as shown in Figure 3(a). The permanent magnets are considered as a constant current source $\Phi$ in the equivalent circuit, as shown in Figure 6. The voltage on the coil $V$ is calculated using Faraday's law:

$$
V=-N \frac{d \Phi_{G}}{d t}=-N \frac{d \Phi_{G}}{d R_{G}} \frac{d R_{G}}{d T(t)} \frac{d T(t)}{d t},
$$

where $N$ is the number of turns of the pickup coil, as optimized in the previous step, $\Phi_{G}$ is the flux through the Galfenol rod, $T(t)$ is the applied stress, and $R_{G}$ is the magnetic reluctance of Galfenol. The term $d R_{G} / d T(t)$ is determined by Galfenol's material property, while the $d T(t) / d t$ term is determined by the input stress wave. The only term in Equation (17) that needs to be optimized is

$$
\frac{d \Phi_{G}}{d R_{G}}=-\frac{R_{c} \Phi}{\left(R_{c}+R_{G}\right)^{2}} .
$$

When the reluctance of the parallel path, $R_{c}$, equals $R_{G}$, the output voltage $V$ reaches a maximum.

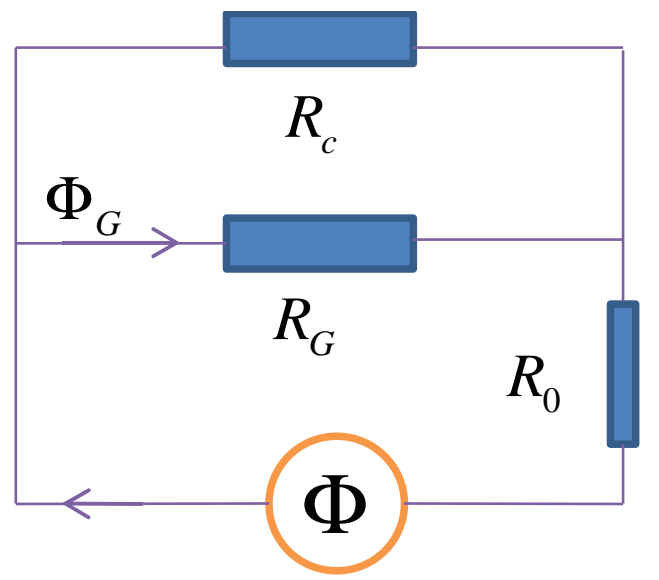

Figure 6. Equivalent circuit for the Galfenol system.

\subsection{Purely resistive load}

\subsubsection{Terfenol-D}

Figure 7 shows the loss factor $\eta$ and average electrical output power $\bar{P}$ with respect to the rod's radius $R_{r}$ and the load resistance $R_{L}$. The maximum output power on the resistive load occurs at around 


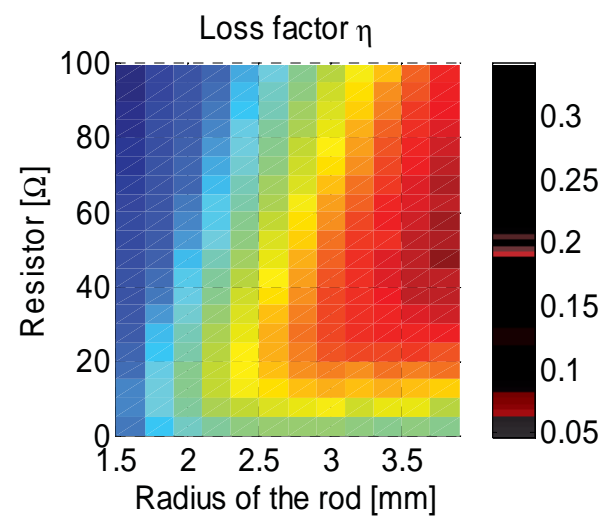

(a)

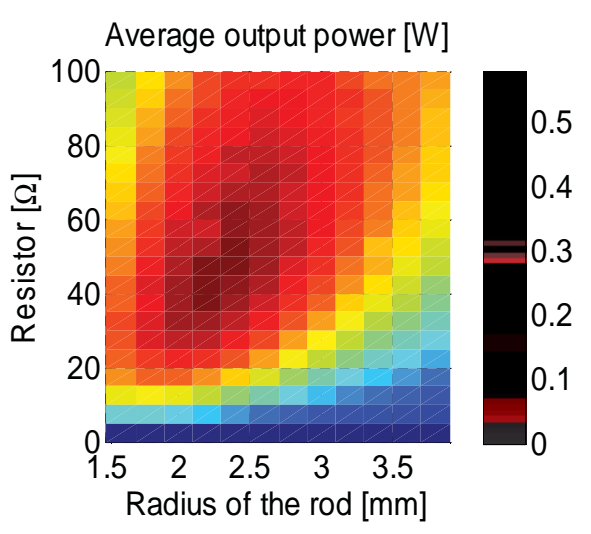

(b)

Figure 7. (a) Loss factor with respect to load resistance and rod radius (Terfenol-D); (b) average electrical output power with respect to load resistance and rod radius (Terfenol-D).

$R_{L}=40 \Omega$ and $R_{r}=2.1 \mathrm{~mm}$, and the maximum output power is $0.58 \mathrm{~W}$. When the pickup coil is shorted, the device cannot output any energy. For small $R_{r}, \bar{P}$ is relatively small, since the $280 \mathrm{~N}$ amplitude input force saturates the material. The inductance of the coil can be estimated by

$$
L_{c}=\frac{\mu N^{2} A}{L}
$$

where $\mu$ is the permeability of the core material, $L$ is the length of the coil, and $A$ is the cross-sectional area of the coil. When $R_{r}$ is large, the impedance of the coil reduces $\bar{P}$. The average electrical output power reaches a maximum when $R_{L}=\sqrt{R_{c}^{2}+\left(L_{c} \omega\right)^{2}}$.

The peak $\eta$ occurs at $R_{L}=55 \Omega$ and $R_{r}=3.9 \mathrm{~mm}$. The maximum $\eta$ is 0.34 . Compared to the $\bar{P}$ surface, the maximum $\eta$ occurs at a relatively low load resistance for the same $R_{r}$. When the loss factor reaches its maximum, the average electrical output power is $0.32 \mathrm{~W}$, which is only $53.5 \%$ of the maximum $\bar{P}$.

\subsubsection{Galfenol}

Figure 8 shows the $\bar{P}$ and $\eta$ surfaces for the Galfenol system. Similar to the Terfenol-D rod, there are peaks in both the loss factor surface and the average electrical output power surface. The maximum $\bar{P}$ and $\eta$ are $0.21 \mathrm{~W}$ and 0.14 , respectively, both of which occurring for a $2 \mathrm{~mm}$ return path thickness. The peak $\bar{P}$ and $\eta$ occur for load resistances of $R_{L}=650 \Omega$ and $R_{L}=500 \Omega$, respectively. Galfenol has a high magnetic permeability, which creates a large inductance $L_{c}$ in the equivalent circuit. Hence, large $R_{L}$ is needed to reach the maximum $\bar{P}$ and $\eta$, and Galfenol has a relatively low average electrical output power and loss factor compared to Terfenol-D.

\subsection{Resistive-capacitive load}

This study considers the case when $R_{L}=+\infty$ (Figure $4(\mathrm{~b})$ ) and $C_{L}$ is varied. To reduce the computational effort, only a Terfenol-D rod with a $3.9 \mathrm{~mm}$ radius and a Galfenol rod with a $2.5 \mathrm{~mm}$ radius are modeled. Figure 9 shows that the loss factor can be improved by impedance matching. The maximum loss factors for Galfenol and Terfenol-D at resonance are 0.59 and 1.83, respectively. But this L-C resonant electric circuit tends to create a narrow loss factor peak in the frequency domain. 


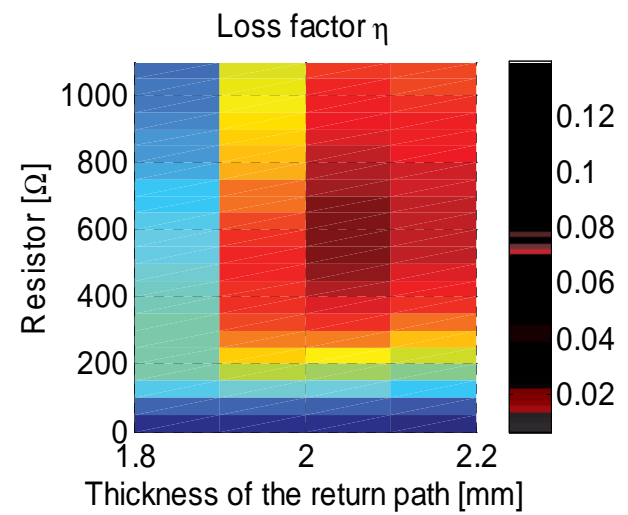

(a)

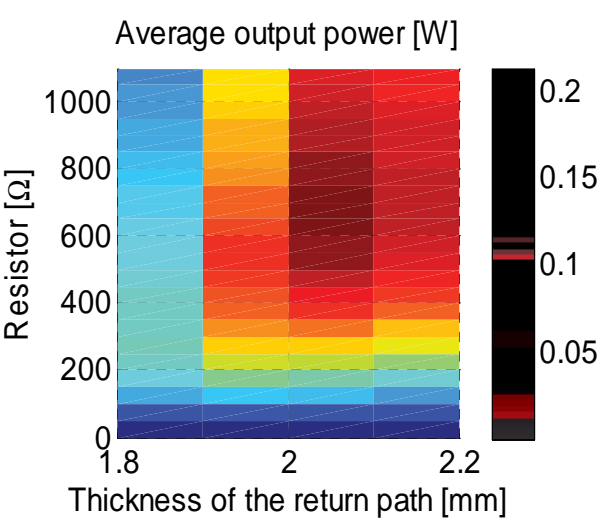

(b)

Figure 8. (a) Loss factor with respect to load resistance and rod radius (Galfenol); (b) average electrical output power with respect to load resistance and rod radius (Galfenol).

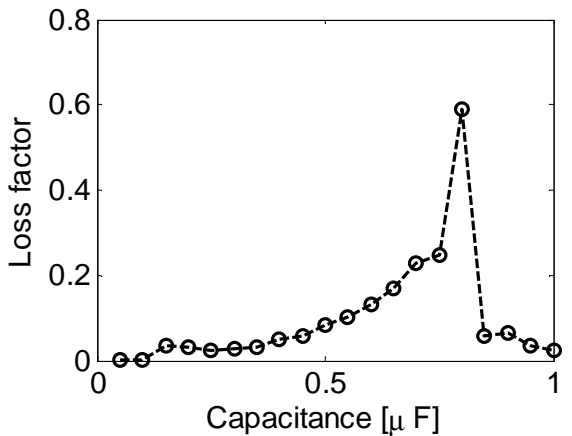

(a)

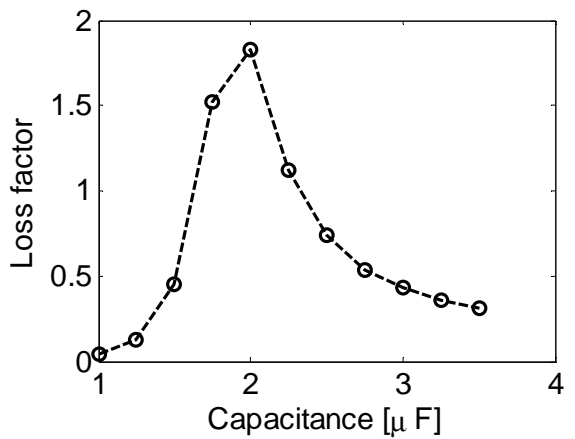

(b)

Figure 9. (a) Loss factor of the Galfenol rod (2.5 mm radius) with respect to varying capacitance; (b) loss factor of the Terfenol-D rod (3.9 $\mathrm{mm}$ radius) with respect to varying capacitance. 


\section{SUMMARY AND FUTURE WORK}

This study provided a modeling framework and design insight for developing a magnetostrictive vibration ring. This device would be mounted within a driveline and transfer mechanical vibration energy to electrical energy to create a damping effect. The work presented focused on optimizing the magnetostrictive elements, which are either Terfenol-D or Galfenol. A parametric study considering loss factor and average electrical output power was conducted using a 2D axisymmetric finite element model. A $750 \mathrm{~Hz}, 280 \mathrm{~N}$ amplitude excitation force was applied to both rods. For purely resistive loads, the maximum loss factors of Galfenol and Terfenol-D are 0.14 and 0.34 , respectively. The maximum average electrical output power $\bar{P}$ for Galfenol and Terfenol-D is $0.21 \mathrm{~W}$ and $0.58 \mathrm{~W}$, respectively. The difference is attributed to the relatively high permeability of Galfenol. This leads to an increased inductance which restricts current variation in the electric circuit. By using an L-C resonant circuit, the maximum loss factors for Galfenol and Terfenol-D increase to 0.59 and 1.83, respectively.

In the next stage of this work, an experiment will be conducted to validate the modeling results. While this work focused on a single frequency, future modeling efforts will investigate the bandwidth of the system. Results from the present study predict that $\bar{P}$ and $\eta$ could be simultaneously maximized by using a copper coil with larger cross section. This improvement will be incorporated in future designs.

\section{ACKNOWLEDGMENTS}

We wish to acknowledge the member organizations of the Smart Vehicle Concepts Center, a National Science Foundation Industry/University Cooperative Research Center (www.SmartVehicleCenter.org) established under NSF Grant IIP-1238286.

\section{REFERENCES}

1. V. M. Asnani, T. L. Krantz, D. C. Delap, and D. B. Stringer, "The Vibration Ring Seedling Fund Phase 1 Final Report," NASA TM-218337, 2014.

2. D. Davino, A. Giustiniani, C. Visone, and A. Adly, "Experimental analysis of vibrations damping due to magnetostrictive based energy harvesting," Journal of Applied Physics 109(7), p. 07E509, 2011.

3. J. Yoo, A. Murray, and A. B. Flatau, "Evaluation of magnetostrictive shunt damper performance using iron (Fe)-gallium (Ga) alloy," in SPIE Smart Structures and Materials $\mathscr{E}$ Nondestructive Evaluation and Health Monitoring, pp. 90573I-90573I, International Society for Optics and Photonics, 2014.

4. T. Ueno and S. Yamada, "Performance of energy harvester using iron-gallium alloy in free vibration," Magnetics, IEEE Transactions on 47(10), pp. 2407-2409, 2011.

5. J. H. Yoo and A. B. Flatau, "A bending-mode Galfenol electric power harvester," Journal of Intelligent Material Systems and Structures 23(6), pp. 647-654, 2012.

6. V. Berbyuk, "Vibration energy harvesting using Galfenol-based transducer," in SPIE Smart Structures and Materials $\mathcal{E}$ Nondestructive Evaluation and Health Monitoring, pp. $86881 \mathrm{~F}-86881 \mathrm{~F}$, International Society for Optics and Photonics, 2013.

7. L. Wang and F. Yuan, "Vibration energy harvesting by magnetostrictive material," Smart Materials and Structures 17(4), p. 045009, 2008.

8. M. B. Moffett, A. E. Clark, M. Wun-Fogle, J. Linberg, J. P. Teter, and E. A. McLaughlin, "Characterization of Terfenol-D for magnetostrictive transducers," Journal of the Acoustical Society of America 89(3), pp. 1448-1455, 1991.

9. A. E. Clark, M. Wun-Fogle, J. B. Restorff, and T. A. Lograsso, "Magnetostrictive properties of Galfenol alloys under compressive stress," Materials Transactions 43(5), pp. 881-886, 2002. 
10. R. Kellogg, A. B. Flatau, A. Clark, M. Wun-Fogle, and T. A. Lograsso, "Temperature and stress dependencies of the magnetic and magnetostrictive properties of $\mathrm{Fe}_{0.81} \mathrm{Ga}_{0.19}$," Journal of Applied Physics 91(10), pp. 7821-7823, 2002.

11. M. Wun-Fogle, J. Restorff, and A. Clark, "Magnetomechanical coupling in stress-annealed Fe-Ga (Galfenol) alloys," Magnetics, IEEE Transactions on 42(10), pp. 3120-3122, 2006.

12. http://www.etrema-usa.com/documents/terfenol.pdf

13. S. Chakrabarti, Modeling of 3D magnetostrictive systems with application to Galfenol and Terfenol$D$ transducers. PhD thesis, The Ohio State University, 2011.

14. Z. Deng and M. J. Dapino, "Characterization and finite element modeling of galfenol minor flux density loops," Journal of Intelligent Material Systems and Structures, p. 1045389X14521703, 2014.

15. E. E. Ungar and E. M. Kerwin Jr, "Loss factors of viscoelastic systems in terms of energy concepts," Journal of the Acoustical Society of America 34(7), pp. 954-957, 1962.

16. ASTM D5992-96(2011), "Standard guide for dynamic testing of vulcanized rubber and rubber-like materials using vibratory methods," ASTM International, West Conshohocken, PA, 2011.

17. Z. Deng and M. J. Dapino, "Modeling and design of Galfenol unimorph energy harvester," in SPIE Smart Structures and Materials \& Nondestructive Evaluation and Health Monitoring, pp. 90572A90572A, International Society for Optics and Photonics, 2014.

18. L. Weng, T. Walker, Z. Deng, M. Dapino, and B. Wang, "Major and minor stress-magnetization loops in textured polycrystalline $\mathrm{Fe}_{81.6} \mathrm{Ga}_{18.4}$ Galfenol," Journal of Applied Physics 113(2), p. $024508,2013$.

19. A. Mahadevan, P. Evans, and M. Dapino, "Dependence of magnetic susceptibility on stress in textured polycrystalline $\mathrm{Fe}_{81.6} \mathrm{Ga}_{18.4}$ and $\mathrm{Fe}_{79.1} \mathrm{Ga}_{21.9}$ Galfenol alloys," Applied Physics Letters $\mathbf{9 6}(1)$, pp. 012502-012502, 2010. 